\title{
Computer Simulations of the Energetics and Siting of n-Alkanes in Zeolites
}

\author{
Berend Smit" \\ Shell Research B.V., Koninklijke/Shell-Laboratorium, Amsterdam, P.O. Box 38000, \\ 1030 BN Amsterdam, The Netherlands
}

\author{
J. Ilja Siepmann ${ }^{\dagger}$ \\ Department of Chemistry, University of Pennsylvania, Philadelphia, Pennsylvania 19104-6323 \\ Received: January 20, 1994; In Final Form: May 13, $1994^{\circ}$
}

\begin{abstract}
The adsorption of the $n$-alkanes, ranging from butane to dodecane, in the zeolites silicalite and (all silica) mordenite has been studied in the zero coverage limit using computer simulations. The heats of adsorption, Henry coefficients, and distribution of the $n$-alkanes over the different zeolite channels are calculated. The calculated heats of adsorption are in good agreement with available experimental data. The calculated heats of adsorption in silicalite show an unexpected alkane length dependence. The incremental heat of adsorption per carbon atom is larger for the longer alkanes than for the shorter alkanes. This observation is attributed to preferential adsorption of the longer alkanes in the straight channels of silicalite. Simulations of this type were made possible through the development of a novel Monte Carlo simulation technique which is orders of magnitude more efficient than the conventional simulation techniques.
\end{abstract}

\section{Introduction}

Zeolites are complex crystalline inorganic polymers of cornersharing $\mathrm{AlO}_{4}$ and $\mathrm{SiO}_{4}$ tetrahedra, which form a three-dimensional framework. This framework has characteristic three-dimensional channels which are accessible to various guest molecules. The large internal surface, the high thermal stability, and the presence of acid sites give zeolites the unique properties that make them an important class of catalytic materials for petrochemical applications. ${ }^{1}$ A catalytic reaction inside a zeolite can be divided into three steps: the adsorption and diffusion of the reactants in the zeolite, the catalytic conversion, and the diffusion and desorption of products out of the zeolite. Of course, this gives only a schematic view of the catalytic processes, but it illustrates the importance of a detailed understanding of the adsorption and diffusion of molecules inside a zeolite at a molecular level. Besides their practical importance, zeolites are also of fundamental interest since they are ideal systems to study the effects of confinement on the properties of adsorbed fluids.

Experimentally, it turned out to be extremely difficult to obtain information on the behavior of adsorbed molecules inside a zeolite at a molecular level. Only recently NMR techniques have been used to study the siting ${ }^{2}$ or diffusion ${ }^{3}$ of adsorbates in zeolites. Detailed information on the behavior of molecules that are of catalytic importance, such as the larger $n$-alkanes, however, is not readily available.

Computer simulations, based on accurate intermolecular potentials, would be an ideal method to fill this gap. Indeed, numerous simulation studies on the adsorption and diffusion of molecules inside a zeolite have been published over the last few years (for a recent overview see ref 4 ). These simulations are limited to relatively small molecules. For these absorbents experimental data is available and these studies have proven that accurate results can be obtained from simulations. At present, the available simulation techniques are limited, due to CPU time restrictions, to relatively small molecules. For example, June $e t$ $a l .5$ used molecular dynamics to study the adsorption and diffusion of butane and hexane in the zeolite silicalite. June et al. pointed

* Author to whom correspondence should be addressed.

+ Permanent address: Department of Chemistry, University of Minnesota, 207 Pleasant St. SE, Minneapolis, MN 55455.

- Abstract published in Advance ACS Abstracts, July 15, 1994. out that a reliable simulation of hexane requires in excess of 1 $\mathrm{ns}$ of physical time to be simulated and therefore requires tens of hours of supercomputer time. In sharp contrast, for xenon a simulation of several picoseconds would already give reliable information. The long relaxation times of the longer alkanes are caused by the relatively slow diffusion in the channels compared to small spherical atoms ${ }^{6}$ and shorter alkanes. ${ }^{7,8}$

Recently, we have developed a novel method, the so-called configurational-bias Monte Carlo technique, to simulate chain molecules.9,10 In this article, we use this technique to study the adsorption of $n$-alkanes in zeolites. Whereas previous methods were limited to the very short alkanes, configurational-bias Monte Carlo can in principle be used for alkanes of arbitrary length.

We continue this article with a description of the models used in this work in section II and in section III with a description of the configurational-bias Monte Carlo method in the context of simulations in zeolites. Thereafter a detailed comparison with the molecular dynamics results of June et al. is given in section IV. In section V some results on the behavior of the alkanes in various zeolites are discussed. Some preliminary results of this work have been published in ref 11 .

\section{Model}

The $n$-alkanes are described with a united-atom model, i.e., $\mathrm{CH}_{3}$ and $\mathrm{CH}_{2}$ groups are considered as single interaction centers. These pseudoatoms in different molecules, or belonging to the same molecule but separated by more than three bonds, interact which each other through a Lennard-Jones potential

$$
u_{i j}^{1 j}=4 \epsilon_{i j}\left[\left(\frac{\sigma_{i j}}{r_{i j}}\right)^{12}-\left(\frac{\sigma_{i j}}{r_{i j}}\right)^{6}\right]
$$

where $r_{i j}$ is the distance between sites $i$ and $j$. The Lennard-Jones potentials were truncated at $13.8 \AA$, and the usual tail corrections have been applied. ${ }^{12}$ The Lennard-Jones parameters used are size parameter $\sigma_{\mathrm{CH}_{3}}=\sigma_{\mathrm{CH}_{2}}=3.93 \AA$ and energy parameter $\epsilon_{\mathrm{CH}_{3}}$ $=114.0 \mathrm{~K}, \epsilon_{\mathrm{CH}_{2}}=47.0 \mathrm{~K}$. The parameters for the unlike interactions have been calculated using the geometric average $\epsilon_{i}$ $=\left(\epsilon_{i} \epsilon_{j}\right)^{1 / 2}$.

The pseudoatoms in a given chain are assumed to be connected by rigid bonds ( $d_{\mathrm{CC}}=1.53 \AA$ ). Bond bending is modeled by a 
TABLE 1: Details on the Zeolite Structures Used in the Simulations, 'no. u' Gives the Number of Unit Cells, 'no. O' the Number of Oxygen Atoms, and $a, b$, and $c$ the Size of the Rectangular Simulation Box

\begin{tabular}{lccccc}
\hline \multicolumn{1}{c}{ zeolite } & no. u & no. O & $a(\AA)$ & $b(\AA)$ & $c(\AA)$ \\
\hline silicalite & 16 & 3072 & 40.140 & 39.840 & 53.680 \\
mordenite & 16 & 1536 & 36.388 & 40.940 & 30.024
\end{tabular}

harmonic potential

$$
u_{\text {bending }}\left(\theta_{i}\right)=1 / 2 k_{\theta}\left(\theta_{i}-\theta_{\text {eq }}\right)^{2}
$$

with $\theta_{\mathrm{eq}}=114^{\circ}$ as the equilibrium angle and with a force constant equal to $k_{\theta}=62500 \mathrm{~K} \mathrm{rad}^{-2} .{ }^{13}$ Changes in the torsional angles are controlled by the Jorgenson potential ${ }^{14}$

$$
\begin{gathered}
u_{\text {torsion }}\left(\phi_{i}\right)=a_{1}\left(1+\cos \phi_{i}\right)+a_{2}\left(1-\cos \left(2 \phi_{i}\right)\right)+ \\
a_{3}\left(1+\cos \left(3 \phi_{i}\right)\right)
\end{gathered}
$$

with $a_{1}=355.03 \mathrm{~K}, a_{2}=-68.19 \mathrm{~K}$, and $a_{3}=791.32 \mathrm{~K}$.

With these parameters the vapor-liquid phase equilibria of the $n$-alkanes can be described accurately over a large temperature range. ${ }^{15}$

In our calculations, we focus on all-silica zeolites. Following Kiselev and co-workers, ${ }^{16}$ the zeolite lattice was assumed to be rigid. Details on the zeolite structures can be found in Table 1 . For alkane adsorption the energetics will be dominated by dispersive interactions. Since the $\mathrm{Si}$ atoms are much smaller than the $\mathrm{O}$ atoms, they have a very small contribution to the energetics and can be ignored in the calculations. In fact, the interactions of the guest molecules with the Si atoms are implicitly accounted for in the effective potential for the interactions with the $\mathrm{O}$ atoms. The dispersive interactions of the $\mathrm{O}$ atoms of the zeolite with the host molecules are described with a LennardJones potential, eq 1. The parameters used are as follows: for the size parameter $\sigma_{\mathrm{CH}_{3}, \mathrm{O}}=\sigma_{\mathrm{CH}_{2}, \mathrm{O}}=3.364 \AA$ and for the energy parameter $\epsilon_{\mathrm{CH}_{3}, \mathrm{O}}=\epsilon_{\mathrm{CH}_{2}, \mathrm{O}}=83.8 \mathrm{~K}$. The potential is truncated at $13.8 \AA$, and the usual tail corrections have been applied. Note that these parameters for the zeolite-host interactions are almost identical to those of June et al. ${ }^{5}$ June et al. used molecular dynamics simulations and therefore used a truncated and shifted Lennard-Jones potential (see section IV). The differences between these potentials are small.

In principle, these simulations can be performed using a vibrating lattice. Demontis et al. ${ }^{17}$ have shown that for dynamical properties it is of importance to take lattice vibrations into account. These vibrations of the zeolite lattice may reduce barriers for diffusion. In this work, we focus on the static properties. In fact, since the Monte Carlo approach is used, information on the dynamics would be impossible to obtain directly. For the static properties, it can be expected that a rigid lattice is a less severe assumption, since barriers, which are essential for diffusion, do not contribute significantly to ensemble averages of most static properties. However, it would be worthwhile to make a detailed comparison to investigate this in more detail in the future.

\section{Computational Aspects}

Before the configurational-bias Monte Carlo method is described, it is instructive to discuss some of the limitations of the conventional Monte Carlo technique. As mentioned in the Introduction, molecular dynamics can be used to calculate properties for molecules which diffuse sufficiently fast such that a representative part of the zeolite is probed during a simulation. If the diffusion is slow, a much longer simulation is required to obtain reliable results.

In principle, Monte Carlo methods can be used to solve this problem, since in a Monte Carlo simulation one does not have to follow the "natural path" of the molecules. For example, one can use a Monte Carlo move in which a particle is moved to an

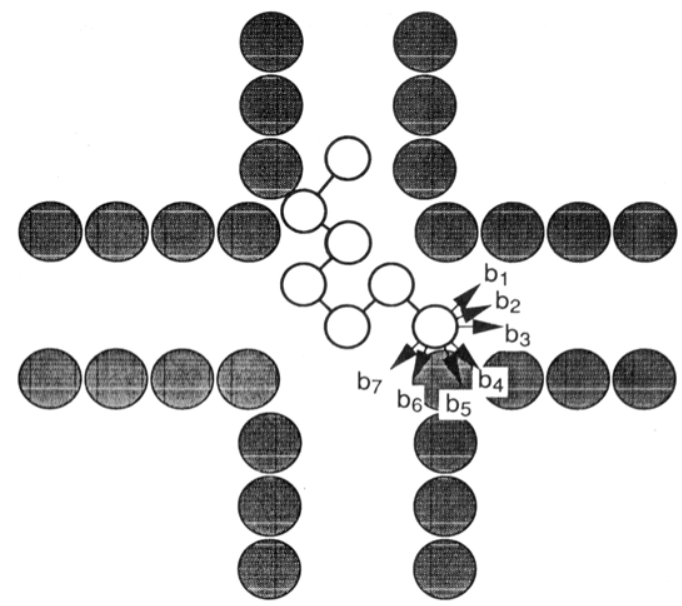

Figure 1. Schematic drawing of the growth of an alkane in a zeolite in a configurational-bias Monte Carlo move. The solid black circles represent the atoms of the zeolite, and the white circles the ones of the alkane. The set of $k$ trial orientations $\left\{\mathbf{b}_{1}, \mathbf{b}_{2}, \ldots, \mathbf{b}_{\mathrm{k}}\right\}$ are indicated by lines.

arbitrary position in the zeolite. For small molecules this is indeed a very efficient method, but it does not work for the longer alkanes. To illustrate this point consider a typical zeolite in which a successful move of a methane molecule, i.e., one which does not result in an overlap with one of the zeolite atoms, has a probability of approximately $10^{-3}$. For ethane this probability will be of the order of $10^{-6}$, and for the longer alkanes this probability is so small that almost none of the attempted moves will be accepted. Configurational-bias Monte Carlo has been developed to solve problems of this kind. Instead of a random insertion, the alkane is "grown" atom by atom such that overlap with the zeolite atoms is avoided. This growth introduces a bias which is exactly removed by adjusting the acceptance rules. ${ }^{9,10,18}$ The configurationalbias Monte Carlo technique has been applied successfully to study self-assembled monolayers ${ }^{19,20}$ and phase equilibria of chain molecules $^{21}$ and $n$-alkanes. ${ }^{15,22.23}$

A. Configurational-Bias Monte Carlo. For systems with strong intramolecular interactions, it is important to take these interactions into account while generating the trial conformation. The potential energy of a given conformation of an $n$-alkane has two contributions: $(i)$ The internal potential energy $\left(U_{\text {int }}\right)$ which includes the bond bending and torsion. (ii) The external potential energy ( $\left.U^{\text {ext }}\right)$ which takes into account the intermolecular interactions and those intramolecular interactions which have not been taken into account in the internal part (the nonbonded Lennard-Jones interactions). Note that this division is to some extent arbitrary and can be optimized for a given application. In what will follow, we refer to a chain without external interactions as an ideal chain.

A new configuration of a randomly selected alkane is generated using the following steps:

1. For the first atom, a random position in the zeolite is selected; the energy of this atom is calculated: $u_{1}{ }^{n}$.

2. For the following atoms, a set of $k$ trial positions is generated. We denote these positions by $\{\mathbf{b}\}=\left(\mathbf{b}_{1}, \mathbf{b}_{2}, \ldots, \mathbf{b}_{k}\right)$ (see Figure 1 ). These positions are distributed on the surface of a sphere. The radius of this sphere is equal to the bond length, and the sphere is centered around the previously inserted atom of the alkane. This set of trial orientations is generated using the internal part of the potential, which results in the following distribution for the lth atom:

$$
p_{l}^{\text {int }}\left(\mathbf{b}_{i}\right) \mathrm{d} \mathbf{b}_{i}=\frac{\exp \left[-\beta u_{l}^{n, \text { int }}\left(\mathbf{b}_{i}\right)\right] \mathrm{d} \mathbf{b}_{i}}{C}
$$

where $\beta=1 / k_{\mathrm{B}} T$ and $C$ is a normalization constant which is not important for the simulations (see below). Note that the probability $p_{I}^{\text {int }}\left(\mathbf{b}_{i}\right)$ depends on which type of atom is being inserted. 
For the second atom, the internal potential energy is zero and as a result the trial positions will be randomly distributed. For the third atom, the internal potential energy includes the bond bending, and for the fourth and higher atoms the internal part includes both the bond bending and torsion (in Appendix A some details on the implementation of this step are given). Of each of these trial positions the external energy is calculated with the atoms of the other molecules (of the zeolite and of other alkanes) and with those atoms of the molecule that are already grown (note that this is done only if $l>4), u^{n, \text { ext }}\left(\mathbf{b}_{j}\right)$, and one of these positions is selected with a probability

$$
p_{l}^{\text {ext }}\left(\mathbf{b}_{j}\right)=\frac{\exp \left[-\beta u_{l}^{n, \text { ext }}\left(\mathbf{b}_{j}\right)\right]}{w^{n, \text { ext }}(l)}
$$

in which

$$
w^{n, \text { ext }}(l)=\sum_{j=1}^{k} \exp \left[-\beta u_{l}^{n, \text { ext }}\left(\mathbf{b}_{j}\right)\right]
$$

(3) after repeating step 2 until the entire alkane of length $M$ has been grown, we calculate

$$
W^{n}=\exp \left[-\beta u^{n, \operatorname{ext}}(1)\right] \prod_{l=2}^{M} w^{n, \text { ext }}(l)
$$

If a new conformation of an alkane molecule has been generated, we proceed by considering the old configuration of the selected alkane molecule: (1) the energy of the first atom is calculated: $u^{\text {o,ext }}(1)$. (2) For the following atoms, the external energy is calculated $u^{\mathrm{o}, \mathrm{ext}}(l)$ and a set of $k-1$ trial orientations is generated with a probability given by eq 4 . Note that also in this case the probability depends on whether the second, third, fourth, or higher atom is considered. Using this set of orientations and the actual position, we calculated for atom $l$

$$
w^{0, e x t}(l)=\exp \left[-\beta u^{0, \operatorname{ext}}(l)\right]+\sum_{j=2}^{k} \exp \left[-\beta u^{0, \operatorname{ext}}\left(\mathbf{b}_{j}\right)\right]
$$

(3) after repeating step 2 until all $M$ atoms of the alkane have been considered, we calculate for the entire molecule

$$
W^{\circ}=\exp \left[-\beta u^{0, \mathrm{ext}}(1)\right] \prod_{l=\frac{2}{2}}^{M} w^{0, \mathrm{ext}}(l)
$$

Finally, the move is accepted with a probability

$$
\operatorname{acc}(0 \rightarrow n)=\min \left(1, W^{n} / W^{\circ}\right)
$$

Note that the factor $W^{\circ}$ depends on the way the old configuration is "retraced". The numerical value of $W^{\circ}$ is different if we start with atom 1 from the value that would be obtained if we start with atom $M$. As a consequence the probability of such a move depends on the way the factor $W^{\circ}$ has been calculated. Such a dependency on the way a move is performed is not uncommon in a Monte Carlo simulation. For example, ${ }^{24}$ consider as a Monte Carlo move the rotation of a molecule around an arbitrary axis. We can perform this Monte Carlo move in two (separate) rotations, and after the first rotation we decide by acceptance/rejection whether to continue with the next rotation. We have the option to perform first a rotation around the $x$-axis followed by one around the $y$-axis, or first around the $y$-axis then around the $x$-axis. In general, the probability that we accept this combined move will depend on the way (first the $x$-axis or first the $y$-axis) we have performed the move. Although such a dependency is at first sight counterintuitive both schemes are correct in the sense that they both lead to desired distribution of configurations. Similarly in the configurational-bias Monte Carlo scheme, both ways - starting with atom 1 or atom $M$-result in the correct distribution of states, as long as both ways occur with equal probability during the simulation. Note that in the case of alkanes the labeling of the terminal groups is completely arbitrary, and therefore it is automatically guaranteed that both ways occur with equal probability during the simulation.

In Appendix B it is shown that acceptance rule (10) indeed removes the bias of the growing process.

An important advantage of the above scheme is that during the insertion step information on the chemical potential can be obtained. It can be shown that the difference of the chemical potential of a chain inside a zeolite and an ideal chain (only having internal interactions) is given by

$$
\mu^{\mathrm{ext}} \equiv \mu-\mu_{\mathrm{IC}}=-\frac{1}{\beta} \ln \left\langle\mathcal{W}^{\mathrm{ext}}\right\rangle
$$

where

$$
\mathcal{W}=W / k^{M-1}
$$

in which $k$ is the number of trial orientations in the configurationalbias Monte Carlo scheme. The subscript IC is used to indicate an ideal chain. The chemical potential of thermodynamic interest is the excess chemical potential $\left(\mu^{\mathrm{ex}}\right)$ which is defined as the difference in chemical potential of the interacting alkane and an alkane in the ideal gas state (denoted by subscript IG)

$$
\mu^{\mathrm{ex}} \equiv \mu-\mu_{\mathrm{IG}}=\mu^{\mathrm{ext}}-\left(\mu_{\mathrm{IG}}-\mu_{\mathrm{IC}}\right)=\mu^{\mathrm{ext}}-\mu_{\mathrm{IG}}{ }^{\mathrm{ext}}
$$

$\mu_{\mathrm{IG}}{ }^{\text {ext }}$ can be estimated from a simulation of one alkane molecule in the ideal gas state using

$$
\mu_{\mathrm{IG}}{ }^{\mathrm{ext}}=-\frac{1}{\beta} \ln \left\langle\mathcal{W}_{\mathrm{IG}}^{\mathrm{ext}}\right\rangle
$$

From the chemical potentials the Henry coefficient can be calculated using

$$
K_{\mathrm{H}} \equiv \beta \exp \left(-\beta \mu^{\mathrm{ex}}\right)=\beta \frac{\left\langle\mathcal{W}^{\text {ext }}\right\rangle}{\left\langle\mathcal{W}_{\mathrm{IG}}^{\text {ext }}\right\rangle}
$$

A separate simulation of an alkane molecule in the ideal gas phase is also necessary to calculate the heat of adsorption. Wood et al. ${ }^{25}$ have shown that in the limit of zero coverage of the zeolite, the heat of adsorption can be calculated using

$$
q_{\mathrm{st}}=\left\langle U_{\mathrm{a}}\right\rangle-\left\langle U_{\mathrm{a}}\right\rangle_{\mathrm{IG}}-k_{\mathrm{B}} T
$$

where $\left\langle U_{\mathrm{a}}\right\rangle_{\mathrm{IG}}$ is the ensemble average of the total energy of an alkane in the ideal gas state.

B. Simulation Details. The simulations were performed in cycles. Each cycle consists of randomly selected attempts to displace a molecule, to rotate a molecule, to regrow part of the molecule, or to regrow the entire molecule. For the latter two moves the configurational-bias technique has been used.

In the displacement step, one of the alkanes is selected at random and given a (small) random displacement. The maximum displacement is chosen such that $50 \%$ of the attempts are accepted.

The rotation step consists of a small rotation of a randomly selected molecule around the space-fixed axes frame. ${ }^{26}$ The center of the rotation is one of the atoms in the middle of the alkane, and the maximum rotation is also chosen such that $50 \%$ of the attempts are accepted.

If a randomly chosen molecule of $M$ atoms is partially regrown, one of the atoms $i \in[2, M-1]$ is randomly selected and it is decided whether to regrow the head or the tail. If the tail is regrown, the algorithm described in the previous section is used to regrow atoms $i-M$; if the head is regrown, the atoms $1-i$ are considered. Note that in this case the first or last atoms of the alkane never change position. This move is very efficient in changing the conformation of an alkane.

If a randomly selected molecule is entirely regrown, all atoms will get a new position. During this step we collect data to calculate the Henry coefficient. All simulations of the alkanes in zeolites were performed using periodic boundary conditions. Due to the 
efficiency of the configurational-bias Monte Carlo moves, equilibrium was already reached after 10-100 Monte Carlo cycles.

To calculate the heat of adsorption and Henry coefficient a separate simulation of the alkane in the ideal gas state is required. In the ideal gas state, a single alkane molecule in an infinitely large simulation box is considered. Because in such a system translation and rotation do not generate new conformations, only configurational-bias Monte Carlo moves were performed. These moves involve regrowing part of the molecule or regrowing the entire molecule.

In our model, the zeolite is assumed to be a rigid lattice. Therefore, the potential energy at a given point inside the zeolite can be calculated à priori and can be used during the simulation. If this is done on a grid, the potential energy at an arbritrary point can be estimated from interpolation. ${ }^{27}$ Because in this work the Monte Carlo method is used exclusively, only the energy has to be calculated with sufficient accuracy (for molecular dynamics the derivatives of the energy have to be accurate as well). A high accuracy of the energy could be obtained using successive polynomial interpolations. ${ }^{28}$ Using this interpolation method with a grid size of $0.18-0.11 \AA$ a gain in CPU time of a factor of $100-300$ could be obtained with an overall error of less than $0.4 \%$ in the energy.

\section{Comparison with Molecular Dynamics Simulations}

To test our simulation technique, a detailed comparison with the molecular dynamics simulations of June et al..$^{5}$ has been made. June et al. studied the adsorption of butane and hexane in silicalite.

The alkane model of June et al. is also a united-atom model with bond bending and torsion. The parameters differ slightly from the ones introduced in the previous section. In the model of June et al., the bond lengths are constrained to $1.53 \AA$. The bond-bending potential is given by eq 2 with $k_{\theta}=62500 \mathrm{~K}$ and an equilibrium angle $\theta_{0}=112^{\circ}$. The torsion potential is of the form used by Ryckaert and Bellemans ${ }^{29}$

$$
u_{\text {torsion }}\left(\phi_{i}\right)=\sum_{k=0}^{5} c_{k} \cos ^{k}\left(\phi_{i}\right)
$$

where $\phi_{i}$ is the dihedral angle. The parameters are $c_{0}=1116 \mathrm{~K}$, $c_{1}=1462 \mathrm{~K}, c_{2}=-1578 \mathrm{~K}, c_{3}=-368 \mathrm{~K}, c_{4}=3156 \mathrm{~K}$, and $c_{5}$ $=-3788 \mathrm{~K}$. The nonbonded interactions are described with a truncated and shifted Lennard-Jones potential. The interactions between the $\mathrm{CH}_{2}$ and $\mathrm{CH}_{3}$ groups are assumed to be identical $(\sigma=3.923 \AA$ and $\epsilon=72 \mathrm{~K})$. The potential is truncated at 13 $\AA$. The interactions of the alkanes with the zeolites are identical to the ones introduced in the previous section.

A. Energetics. Using the Monte Carlo method as described in the previous section, simulations of butane and hexane in silicalite have been performed using identical systems and models as in the molecular dynamics simulations of June et al..$^{5}$ In Table 2 the average energies are compared showing that the agreement between the two methods is excellent.

In Table 3 the results for the isosteric heat of adsorption and the Henry coefficient are compared. The results for the isosteric heat of adsorption and the Henry coefficient agree less than one would expect on the basis of the results of Table 2. This suggests that there may be a systematic difference in the calculation of the Henry coefficient of June et al. and our calculations. June et al. calculated the Henry coefficient using

$$
K_{\mathrm{H}}=\frac{M_{\mathrm{a}}}{N_{\mathrm{a}} \rho_{\mathrm{z}}} \beta \frac{\int \mathrm{d} \Gamma \exp [-\beta U(\Gamma)]}{\int \mathrm{d} \Gamma \exp \left[-\beta U_{\mathrm{IG}}(\Gamma)\right]}
$$

where $\Gamma$ denotes the position of the atoms of an alkane molecule, $M_{\mathrm{a}}$ is the molecular mass of the adsorbate, $\rho_{\mathrm{z}}$ the density of the zeolite, and $N_{\mathrm{a}}$ Avogadro's number. The subscript IG indicates that the interactions are those of an alkane in the ideal gas state.
TABLE 2: Comparison of the Molecular Dynamics Results of June et al.s (Denoted with “J") with the Results of Our Monte Carlo Simulations (Denoted with " $O$ "). $T$ Is the Temperature, $\left\langle U^{\text {s2 }}\right\rangle$ the Mean Sorbate-Zeolite Energy, $\left\langle U^{\text {ms }}\right\rangle$ the Sorbate-Sorbate Energy, $\left\langle U^{b}\right\rangle$ the Bond-Bending Energy, $\left\langle U^{*}\right\rangle$ the Torsion Energy, and $\left\langle U^{*}\right\rangle$ the Nonbonded Energy. All Simulations Were Performed with 64 Molecules (4 Sorbate Molecules per Unit Cell of Silicalite). The Simulation Cell Size was $40.14 \times 39.64 \times 53.68 \AA$. The Subscripts Give the Accuracy of the Results, so 3.5. Means

\begin{tabular}{|c|c|c|c|c|c|c|}
\hline & $\begin{array}{c}T \\
(\mathrm{~K})\end{array}$ & $\begin{array}{c}\left\langle U^{\mathrm{sz}}\right\rangle \\
(\mathrm{kJ} / \mathrm{mol})\end{array}$ & $\begin{array}{c}\left\langle U^{\mathrm{ss}}\right\rangle \\
(\mathrm{kJ} / \mathrm{mol})\end{array}$ & $\begin{array}{c}\left\langle U^{\infty}\right\rangle \\
(\mathrm{kJ} / \mathrm{mol}) \\
\end{array}$ & $\begin{array}{c}\langle U\rangle \\
(\mathrm{kJ} / \mathrm{mol})\end{array}$ & $\begin{array}{c}\left\langle U^{3}\right\rangle \\
(\mathrm{kJ} / \mathrm{mol})\end{array}$ \\
\hline \multicolumn{7}{|c|}{ Butane } \\
\hline $\begin{array}{l}\text { J } \\
\text { O }\end{array}$ & $\begin{array}{l}289 \\
289\end{array}$ & $\begin{array}{l}-45.6_{2} \\
-44.86_{6}\end{array}$ & $\begin{array}{l}-0.48_{8} \\
-0.42_{1}\end{array}$ & $\begin{array}{l}2.459 \\
2.40_{1}\end{array}$ & $\begin{array}{l}2.1_{2} \\
2.05_{2}\end{array}$ & \\
\hline \multicolumn{7}{|c|}{ Hexane } \\
\hline $\begin{array}{l}\text { J } \\
\text { O }\end{array}$ & $\begin{array}{l}308.1 \\
308.1\end{array}$ & $\begin{array}{l}-66.3_{2} \\
-65.29_{8}\end{array}$ & $\begin{array}{l}-0.9_{2} \\
-0.83_{5}\end{array}$ & $\begin{array}{l}5.1_{2} \\
5.09_{3}\end{array}$ & $\begin{array}{l}6.5_{2} \\
6.33_{\mathrm{s}}\end{array}$ & $\begin{array}{l}-1.12_{2} \\
-1.11_{1}\end{array}$ \\
\hline
\end{tabular}
$3.5 \pm 0.4$.

TABLE 3: Comparison with the Molecular Dynamics Results of June et $a .^{5}$ of the Isosteric Heat of Adsorption $q_{s t}$ and the Henry Coefficient $\left\langle K_{H}\right\rangle$ (See Also Table 2)

\begin{tabular}{cccc}
\hline & $T(\mathrm{~K})$ & $q_{\mathrm{st}}(\mathrm{kJ} / \mathrm{mol})$ & $\left\langle K_{\mathrm{H}}\right\rangle((\mathrm{mg} / \mathrm{g}) / \mathrm{atm})$ \\
\hline $\mathrm{J}$ & 393 & 48.3 & $1612_{32}$ \\
$\mathrm{O}$ & 393 & $46.3_{1}$ & $1003_{60}$
\end{tabular}

TABLE 4: Henry Coefficient of Butane in Silicalite. Comparison of the Results of June et $a .^{5}$ (Denoted with " J") with the Results of Our Monte Carlo Simulations (Denoted with " 0 "). " $O_{\text {shift }}$ are the calculations with the Truncated and Shifted Potential, and " $O_{\text {no-shift }}$ Those with the Truncated but $N o t$ Shifted Potential. $T$ Is the Temperature, $q_{\text {st }}$ Is the Isosteric Heat of Adsorption, and $\left\langle K_{H}\right\rangle$ Is the Henry Coefficient. For Comparison the Experimental Data Have Been Added

\begin{tabular}{lccc}
\hline & $T(\mathrm{~K})$ & $q_{\mathrm{Bt}}(\mathrm{kJ} / \mathrm{mol})$ & $\left\langle K_{\mathrm{H}}\right\rangle((\mathrm{mg} / \mathrm{g}) / \mathrm{atm})$ \\
\hline $\mathrm{J}$ & 393 & 48.3 & $1612_{32}$ \\
$\mathrm{O}_{\text {shift }}$ & 393 & $46.3_{1}$ & $1003_{60}$ \\
$\mathrm{O}_{\text {no-shift }}$ & 393 & $47.1_{3}$ & $1338_{90}$ \\
$\exp ^{47}$ and Table 8 & 393 & 50 & $1500_{50}$
\end{tabular}

For small molecules the parition functions in eq 18 can be calculated via Monte Carlo integration. ${ }^{28}$ We have repeated these calculations for methane, ethane, propane, butane, and pentane and compared them with the results based on eq 15 . Both methods gave identical results. In fact, since the two methods are formally equivalent, a difference would indicate an error in one of the two codes. However, these calculations do not resolve the differences between the results of June et al. and our calculations.

June et al. used Monte Carlo integration ${ }^{28}$ to calculate the Henry coefficient. In order to be consistent with the molecular dynamics potential, we used a truncated and shifted potential. It is not always realized that the shift of the potential can have a significant effect on the results. ${ }^{30,31}$ To test the effects of truncation, we have performed some calculations for butane with a truncated but not shifted potential. The results shown in Table 4 suggest that at least part of the differences may be attributed to this.

The consistency of the results can also be tested via a thermodynamic relation which, in the limit of zero coverage, links the Henry coefficient to the heat of adsorption via

$$
\frac{q_{\mathrm{st}}}{N_{\mathrm{a}}}=-\frac{\partial \ln \left(K_{\mathrm{H}}\right)}{\partial\left(1 / k_{\mathrm{B}} T\right)}
$$

In Figure 2 the Henry constant of butane in silicalite, as calculated using eq 15 , is shown as a function of the reciprocal temperature. If we assume that the isosteric heat of adsorption is constant in this temperature range, eq 19 can be used to fit the data. The resulting heat of adsorption is $q_{\mathrm{st}}=46.0 \pm 0.4 \mathrm{~kJ} / \mathrm{mol}$. This 


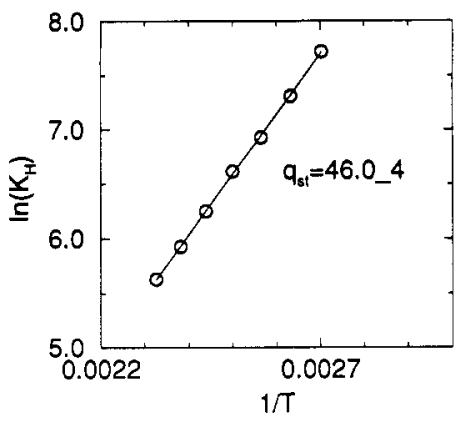

Figure 2. Isosteric heat of adsorption $(\mathrm{kJ} / \mathrm{mol})$ of butane calculated from the temperature $(T)$ dependence of the Henry coefficient $\left(K_{\mathrm{H}}\right)$.

result is in perfect agreement with a direct calculation of the heat of adsorption at $T=400 \mathrm{~K}$ which gave $46.1 \pm 0.2 \mathrm{~kJ} / \mathrm{mol}$.

From the above comparison, we conclude that the agreement of our calculations with the molecular dynamics results of June et al. is very good. Only for the Henry coefficients, which were not calculated using molecular dynamics by June et al., is the agreement less than satisfactory. A complete explanation of these differences could not be obtained. However, several tests have been applied successfully to check the consistency of our calculations of the Henry coefficient. Based on these tests we conclude that our method indeed yields reliable results.

B. Efficiency. It is instructive to compare the efficiency of molecular dynamics with our Monte Carlo simulations. At this point it is important to note that Monte Carlo can only yield static properties; if one is interested in dynamics, Monte Carlo cannot be used and one has to rely on molecular dynamics simulations.

An important question in computer simulations is whether they have been continued sufficiently long. For an alkane in a zeolite June et al. showed that the slowest relaxation is the diffusion of the molecules from one channel into another. For example, hexane has to undergo conformational changes (involving multiple crossings of the trans/gauche barrier) while passing from a straight channel into a zigzag channel. June et al. observed that this relaxation time is of the order of 500 ps. This implies that a reliable simulation, i.e., one which would ideally span at least 10 relaxation times, ${ }^{12}$ should extend over at least $1000-5000$ ps. Since this requires $10-50 \mathrm{~h}$ of Cray (Y-MP/864) time, it can be understood that alkanes larger than hexane are extremely costly to study using the molecular dynamics technique.

The pores of the zeolite impose an orientation on the molecule (see section $\mathrm{V}$ for details). The orientation of the end to end vector can therefore be used to monitor the diffusion of the alkanes. ${ }^{5}$ June et al. used the first Legendre polynomial to characterize the correlations. In a Monte Carlo simulation, however, $P_{1}(t)$ would rapidly approach zero by relabeling of the atoms (i.e., changing the head and tail). Note that in a molecular dynamics simulation such a move would take many time steps since the molecule has to diffuse out of the channel, rotate, and come back again. In a Monte Carlo study it is therefore more appropriate to monitor the second-order Legendre polynomial $P_{2}(t)$

$$
P_{2}(t)=\left\langle\frac{3}{2}(\hat{\mathbf{R}}(0) \cdot \hat{\mathbf{R}}(t))^{2}-\frac{1}{2}\right\rangle
$$

where $\hat{\mathbf{R}}$ is the normalized end to end vector. Note that if the molecules align $P_{2}(\infty)=1$, if the molecules are randomly distributed $P_{2}(\infty)=0$, and if the molecules are on average perpendicular $P_{2}(\infty)=-0.5$. If we assume that the molecules are perpendicular to each other in the two different channels and that the contribution of the intersection is negligible, $P_{2}(\infty)$ can

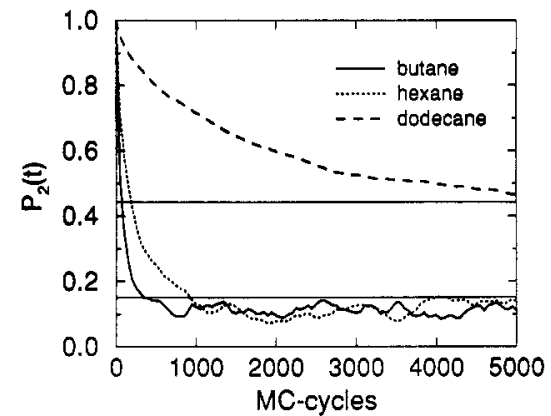

Figure 3. Orientation correlation functions of butane (solid line), hexane (dotted line), and dodecane (dashed line). The horizontal lines are the estimates of $P_{2}(\infty)$ using eq 21 .

TABLE 5: Distribution of Alkanes over the Various Channels (Zigzag, Straight, and Intersection) of Silicalite at $T=298 \mathrm{~K}$

\begin{tabular}{lccccccc}
\hline$n$ & zigzag & straight & intersection & $n$ & zigzag & straight & intersection \\
\hline $\mathrm{C}_{4}$ & 0.50 & 0.42 & 0.08 & $\mathrm{C}_{9}$ & 0.31 & 0.65 & 0.04 \\
$\mathrm{C}_{5}$ & 0.49 & 0.44 & 0.07 & $\mathrm{C}_{10}$ & 0.18 & 0.78 & 0.03 \\
$\mathrm{C}_{6}$ & 0.45 & 0.49 & 0.06 & $\mathrm{C}_{11}$ & 0.18 & 0.79 & 0.04 \\
$\mathrm{C}_{7}$ & 0.41 & 0.54 & 0.05 & $\mathrm{C}_{12}$ & 0.20 & 0.77 & 0.03 \\
$\mathrm{C}_{8}$ & 0.36 & 0.59 & 0.05 & & & &
\end{tabular}

be estimated from

$$
P_{2}(\infty) \approx p_{\text {str }}\left(1.0 p_{\mathrm{str}}-0.5 p_{z z}\right)+p_{z z}\left(-0.5 p_{\mathrm{str}}+1.0 p_{z z}\right)
$$

where $p_{\text {str }}$ is the probability that a molecule is in a straight channel. The equilibrium distribution over the channels in silicalite is given in Table 5. In Figure 3, the orientation correlation functions of butane, hexane, and dodecane are shown.

From Figure 3 it follows that the correlation decay "time" for butane is of the order of 100 Monte Carlo cycles, for hexane 300 cycles, and for dodecane 3000 cycles. A reliable simulation would therefore require at least $5-10$ relaxation times, i.e., 3000 cycles for hexane. Note that it took June et al. 50 h of Cray (Y-MP/ 864 ) time to simulate only 2 relaxation times. In our program, 3000 Monte Carlo cycles take 2-6 min on a workstation (Silicon Graphics, Personal Iris). ${ }^{32}$ This significant increase in efficiency allows us to study the adsorption of the longer alkanes in various zeolites.

\section{Adsorption of n-Alkanes}

We have used the configurational-bias Monte Carlo technique to study the adsorption of $\mathrm{C}_{4}-\mathrm{C}_{12}$ in various zeolites. The details of the zeolite structures are shown in Table 1. The simulations are performed in the limit of infinite low coverage, i.e., one alkane molecule. Some details of the simulations are given in Table 6.

In Table 7 the calculated heats of adsorption and Henry coefficients of the alkanes in various zeolites are presented. The values in the ideal gas state, which are required to calculate the heats of adsorption and Henry coefficients (see eqs 15 and 16), are presented as well.

A. Silicalite. The heats of adsorption of the various $n$-alkanes in silicalite and ZSM5 have been determined experimentally using calorimetry, ${ }^{33-39}$ from adsorption isotherms, ${ }^{34,39-46}$ or using gas chromatography, ${ }_{4}^{4748}$ In Table 8, some of the experimental data for silicalite and ZSM5 with a high $\mathrm{Si} / \mathrm{Al}$ ratio are compiled. Although some scatter of the experimental data as obtained from the various techniques exists, the overall consistency is good. The data that are used in the comparison with the simulation results are also shown in Table 8.

In Figure 4, the calculated heats of adsorption of the alkanes in silicalite are compared with the experimental data. The chain length dependence of the Henry coefficient is shown in Figure 5. Dubinin et al. ${ }^{38}$ showed, using experimental data of $\mathrm{C}_{2}-\mathrm{C}_{5}$, that the heat of adsorption increases with $10.0 \mathrm{~kJ} / \mathrm{mol}$ per $\mathrm{CH}_{2}$ 
TABLE 6: Details of the Simulations. The Simulations were Performed with One Alkane molecule. Cycles Gives the Total Number of Monte Carlo Cycles. During One Cycle the

Probabilities of a Translation, Rotation, Partly Regrowing, or Regrowing of the Entire Molecule were $10 \%, 10 \%, 20 \%$, and $60 \%$, Respectively. The Number of Trial Orientations $k$ Was 6. The Probability (Percentage) of a Successful Regrowing Move in Silicalite and Mordenite for $T=298 \mathrm{~K}$ Are Indicated by suc.(Sil.)' and suc.(Mor.)', Respectively. The CPU Time (in hh:mm) Is for a Silicon Graphics Personal Iris 4D/35 Workstation for the Simulation in Silicalite

\begin{tabular}{ccccc}
\hline$n$ & cycles & suc.(Sil.) & suc.(Mor.) & CPU time \\
\hline $\mathrm{C}_{4}$ & $10^{6}$ & 1.11 & 2.21 & $7: 04$ \\
$\mathrm{C}_{5}$ & $10^{6}$ & 0.66 & 1.21 & $10: 21$ \\
$\mathrm{C}_{6}$ & $10^{6}$ & 0.48 & 0.89 & $13: 33$ \\
$\mathrm{C}_{7}$ & $10^{6}$ & 0.30 & 0.63 & $16: 16$ \\
$\mathrm{C}_{8}$ & $10^{6}$ & 0.20 & 0.48 & $18: 50$ \\
$\mathrm{C}_{9}$ & $10^{6}$ & 0.11 & 0.34 & $21: 03$ \\
$\mathrm{C}_{10}$ & $10^{6}$ & 0.05 & 0.22 & $23: 25$ \\
$\mathrm{C}_{11}$ & $10^{6}$ & 0.04 & 0.20 & $25: 29$ \\
$\mathrm{C}_{12}$ & $10^{6}$ & 0.02 & 0.15 & $27: 07$
\end{tabular}

TABLE 7: Total Energy $\langle U\rangle_{a}$, Sorbate-Zeolite energy $\langle U\rangle_{2}$, (External) Chemical Potential ( $\left.\mu^{\text {ext }}\right)$, Isosteric Heat of Adsorption $q_{i s}$, and Henry Coefficient $K_{\mathrm{H}}$ of the $n$-Alkanes in the Ideal Gas Phase and in the Zeolites Silicalite and Mordenite

\begin{tabular}{|c|c|c|c|c|c|}
\hline$n$ & $\begin{array}{l}\langle U\rangle_{\mathrm{a}} \\
(\mathbf{K})^{2}\end{array}$ & $\begin{array}{c}\langle U\rangle_{2} \\
(\mathrm{~K})\end{array}$ & $\begin{array}{l}\mu^{\text {ext }} \\
(\mathrm{K})\end{array}$ & $\begin{array}{c}q_{\text {is }} \\
(\mathrm{kJ} / \mathrm{mol})\end{array}$ & $\begin{array}{c}\ln K_{\mathrm{H}} \\
\ln ((\mathrm{mg} / \mathrm{g}) / \mathrm{atm})\end{array}$ \\
\hline $\begin{array}{l}C_{4} \\
C_{5} \\
C_{6} \\
C_{7} \\
C_{8} \\
C_{9} \\
C_{10} \\
C_{11} \\
C_{12}\end{array}$ & $\begin{array}{r}608_{17} \\
943_{15} \\
1329_{32} \\
1707_{40} \\
2067_{26} \\
2444_{20} \\
2817_{33} \\
3227_{27} \\
3614_{27}\end{array}$ & & $\begin{array}{c}\text { Ideal Gas } \\
0 \\
-66.7_{3} \\
-119.4_{5} \\
-167.5_{8} \\
-216.8_{6} \\
-271.2_{8} \\
-321.9_{9} \\
-376_{1} \\
-430_{1}\end{array}$ & & \\
\hline $\begin{array}{l}C_{4} \\
C_{5} \\
C_{6} \\
C_{7} \\
C_{8} \\
C_{9} \\
C_{10} \\
C_{11} \\
C_{12}\end{array}$ & $\begin{array}{l}-4955_{33} \\
-5938_{70} \\
-6824_{115} \\
-7794_{81} \\
-8783_{81} \\
-9819_{207} \\
-11148_{186} \\
-12280_{212} \\
-13372_{267}\end{array}$ & $\begin{array}{c}-5500_{27} \\
-6793_{48} \\
-8029_{46} \\
-9308_{52} \\
-10597_{53} \\
-11929_{83} \\
-13407_{107} \\
-14830_{128} \\
-16210_{127}\end{array}$ & $\begin{array}{l}\text { Silicalite } \\
-3325_{24} \\
-4334_{20} \\
-5386_{24} \\
-6391_{16} \\
-7458_{24} \\
-8473_{15} \\
-9601_{123} \\
-10522_{123} \\
-11677_{167}\end{array}$ & $\begin{array}{l}48.7_{4} \\
59.7_{7} \\
70_{1} \\
82_{1} \\
93_{1} \\
104_{2} \\
119_{2} \\
131_{2} \\
144_{2}\end{array}$ & $\begin{array}{l}11.44_{1} \\
14.82_{1} \\
18.35_{1} \\
21.71_{1} \\
25.26_{1} \\
28.59_{1} \\
32.31_{1} \\
35.32_{1} \\
39.10_{1}\end{array}$ \\
\hline $\begin{array}{l}C_{4} \\
C_{5} \\
C_{6} \\
C_{7} \\
C_{8} \\
C_{9} \\
C_{10} \\
C_{11} \\
C_{12}\end{array}$ & $\begin{array}{l}-3594_{20} \\
-4364_{48} \\
-5056_{50} \\
-5792_{59} \\
-6565_{49} \\
-7249_{114} \\
-8028_{65} \\
-8814_{158} \\
-9550_{222}\end{array}$ & $\begin{array}{l}-4233_{9} \\
-5297_{19} \\
-6362_{12} \\
-7445_{21} \\
-8513_{28} \\
-9578_{38} \\
-10660_{29} \\
-11743_{38} \\
-12817_{64}\end{array}$ & $\begin{array}{l}\text { Mordenite } \\
-2766_{27} \\
-3618_{33} \\
-4509_{51} \\
-5429_{92} \\
-6325_{47} \\
-7200_{64} \\
-8088_{102} \\
-9078_{154} \\
-9951_{115}\end{array}$ & $\begin{array}{c}37.4_{3} \\
46.6_{5} \\
55.6_{7} \\
64.8_{8} \\
74.2_{6} \\
83_{1} \\
93_{1} \\
103_{2} \\
112_{2}\end{array}$ & $\begin{array}{l}9.56_{1} \\
12.41_{1} \\
15.40_{1} \\
18.48_{2} \\
21.45_{1} \\
24.32_{1} \\
27.24_{1} \\
30.47_{2} \\
33.30_{1}\end{array}$ \\
\hline
\end{tabular}

group. This is in good agreement with our calculations which give an increase of $11 \mathrm{~kJ} / \mathrm{mol}$ per $\mathrm{CH}_{2}$ group. Interestingly, we observe a change in slope for the longer alkanes. For alkanes longer than heptane we observe an increase of $13 \mathrm{~kJ} / \mathrm{mol}$ per $\mathrm{CH}_{2}$ group. This change in slope turned out to have a simple molecular explanation.

Figure 6 shows a schematic picture of the silicalite structure. The zeolite structure has of two sets of pores, one that forms the straight channels which are parallel to the $y$-axis and the zigzag channels which are parallel to the $x$-axis. As mentioned in the previous section, the channels of silicalite impose an orientation of the alkane molecule, which can be analyzed by calculating the orientation of the end to end vector of the alkane. If the orientation of the end to end vector is parallel to the $x$-axis, the molecule is located in the zigzag channel; if it is parallel to the $y$-axis the

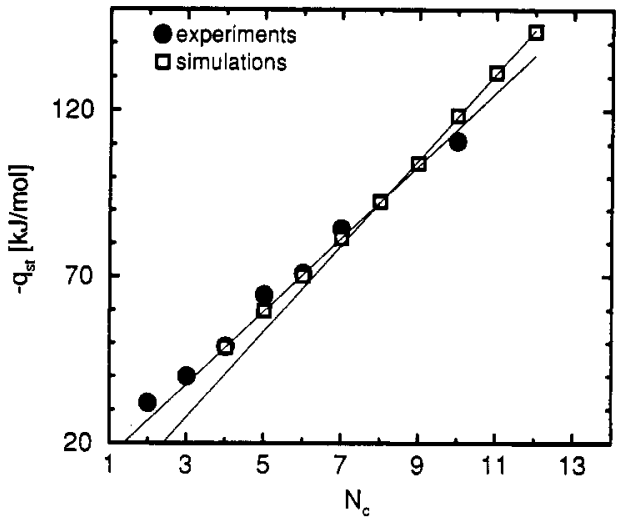

Figure 4. Heats of adsorption $q_{\mathrm{st}}$ of the alkanes in silicalite as a function of the chain length $N_{\mathrm{c}}$. The solid lines are linear fits to the data, which give a slope of $11.0 \mathrm{~kJ} / \mathrm{mol}$ per carbon atom for $\mathrm{C}_{4}-\mathrm{C}_{8}$, and $12.9 \mathrm{~kJ} / \mathrm{mol}$ per carbon atom for $\mathrm{C} 8-\mathrm{C}_{12}$. The experimental data are from Table 8.

TABLE 8: The Experimental Heat of Adsorption ( $\left.q_{\mathrm{st}}\right)$ of Various Alkanes in Silicalite/ZSM5. The Experimental Methods (meth) are; (a) Adsorption Isotherms, (c) Calorimetric, and (g) Gas Chromatography. The Selected Value of the Heats of Adsorption Are Given below sel

\begin{tabular}{|c|c|c|c|c|c|}
\hline $\begin{array}{c}T \\
(\mathrm{~K})\end{array}$ & $\begin{array}{l}\mathrm{Si} / \mathrm{Al} \\
\text { ratio }\end{array}$ & $\begin{array}{c}q_{\mathrm{st}} \\
(\mathrm{kJ} / \mathrm{mol})\end{array}$ & meth & ref & $\operatorname{sel}(\mathrm{K})$ \\
\hline $\begin{array}{l}298 \\
300 \\
300 \\
300 \\
301 \\
318\end{array}$ & $\begin{array}{l}\infty \\
\infty \text { (Linde S-115) } \\
\infty \\
\infty \\
\infty \\
1230\end{array}$ & $\begin{array}{l}\text { Ethane } \\
30.5 \\
32.8 \\
29.9 \\
31 \\
34 \\
30\end{array}$ & $\begin{array}{l}\mathrm{a} \\
\mathrm{a} \\
\mathrm{g} \\
\mathrm{a} \\
\mathrm{c} \\
\mathrm{a}\end{array}$ & $\begin{array}{l}43 \\
41 \\
55 \\
34 \\
33 \\
44\end{array}$ & 32 \\
\hline $\begin{array}{l}300 \\
298 \\
300 \\
325 \\
318 \\
423\end{array}$ & $\begin{array}{l}\infty \\
\infty \\
\infty \\
\infty(\text { Linde S-115) } \\
1230 \\
\infty\end{array}$ & $\begin{array}{l}\text { Propane } \\
42.2 \\
38_{1} \\
40.7 \\
39.9 \\
40 \\
36.5\end{array}$ & $\begin{array}{l}\mathrm{a} \\
\mathrm{g} \\
\mathrm{a} \\
\mathrm{a} \\
\mathrm{g}\end{array}$ & $\begin{array}{l}46 \\
43 \\
55 \\
41 \\
44 \\
47\end{array}$ & 40 \\
\hline $\begin{array}{l}300 \\
300 \\
301 \\
325 \\
400 \\
423\end{array}$ & $\begin{array}{l}\infty \\
\infty \\
\infty \\
\infty(\text { Linde S-115) } \\
\infty \\
\infty\end{array}$ & \begin{tabular}{l}
\multicolumn{1}{c}{ Butane } \\
51 \\
50.4 \\
$54.8_{1}$ \\
48.3 \\
48 \\
49.5
\end{tabular} & $\begin{array}{l}\mathrm{c} \\
\mathrm{g} \\
\mathrm{c} \\
\mathrm{a} \\
\mathrm{g} \\
\mathrm{g}\end{array}$ & $\begin{array}{l}34 \\
55 \\
35,36,33 \\
41 \\
48 \\
47\end{array}$ & 50 \\
\hline 303 & $\infty$ & $\begin{array}{l}\text { Pentane } \\
64.5_{4}\end{array}$ & c & 37,38 & 64.5 \\
\hline $\begin{array}{l}300 \\
300 \\
300\end{array}$ & $\begin{array}{l}\infty \\
\infty \\
\infty\end{array}$ & $\begin{array}{l}\text { Hexane } \\
71_{4} \\
71.5_{1} \\
71\end{array}$ & $\begin{array}{l}a \\
c \\
c\end{array}$ & $\begin{array}{l}40 \\
56,39 \\
34\end{array}$ & 71 \\
\hline 303 & $\infty$ & $\begin{array}{l}\text { Heptane } \\
84.5_{3}\end{array}$ & c & 37,38 & 85 \\
\hline $\begin{array}{l}300 \\
303\end{array}$ & $\infty$ & $\begin{array}{l}\text { Decane } \\
112 \\
110.5_{4}\end{array}$ & a & $\begin{array}{l}34 \\
39\end{array}$ & 111 \\
\hline
\end{tabular}

molecule is in the straight channel, and otherwise the molecule is in the intersection.

The distribution of the various alkanes over the straight channel, zigzag channel, and intersection as a function of carbon number is shown in Figure 7. For the short alkanes, the probability of being in the zigzag channel is nearly equal to the probability of being in the straight channel. For the larger alkanes $\left(>C_{8}\right)$, the straight channel is favored above the zigzag channel. This is also illustrated in Figure 8. These figures show a distribution of the alkanes over the various channels. At regular intervals during the simulations, the end to end vector of the alkane molecule is calculated and depending on its orientation a color is selected: 


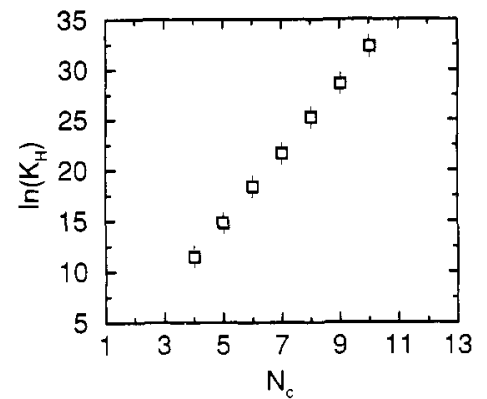

Figure 5. Henry coefficients $K_{H}$ of the alkanes in silicalite as a function of the chain length $N_{\mathrm{c}}$.

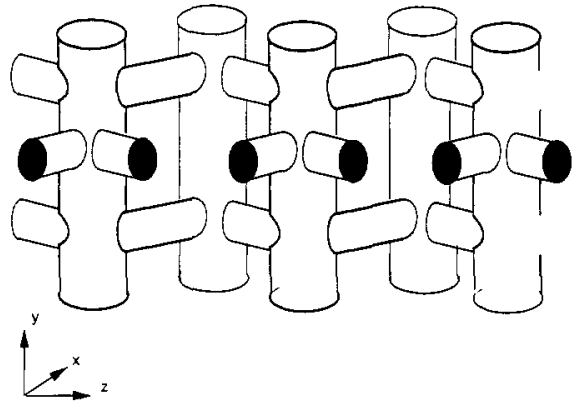

Figure 6. Schematic drawing of the pore structure of silicalite.

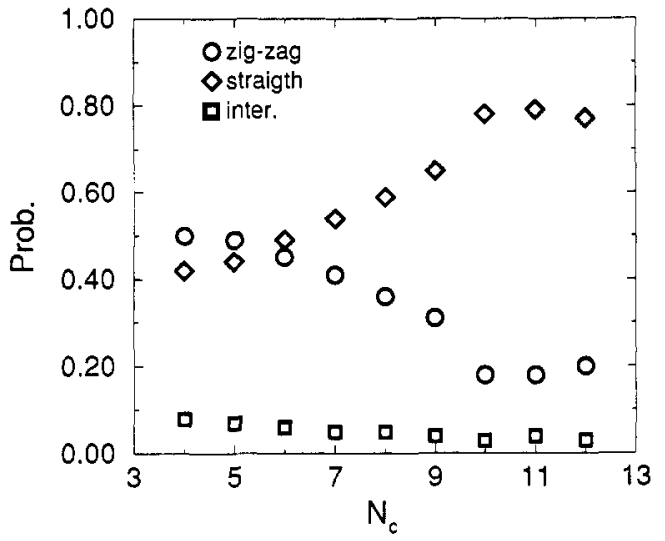

Figure 7. Probability of finding an $n$-alkane in the straight channel, zigzag channel, or intersection of silicalite as a function of carbon number $N_{\mathrm{c}}$.

blue for the intersection, gray for the zigzag channel, and magenta for the straight channel. Next the middle of the bond between the two middle atoms is determined, and a dot in the selected color is drawn. The figures demonstrate that the selection of channels by the orientation of the end to end vector characterizes the location of the alkane correctly. The density of the dots is a measure of the probability of finding an alkane in a particular section of the zeolite. These graphs show that during the simulation all parts of the zeolites have been visited. This is important evidence that the simulations are reliable. It is interesting to compare the probability distribution of butane, Figure 8 (top) with the one of decane Figure 8 (bottom). Whereas the plots of butane show an equal density of points in the straight and zigzag channels, decane has a significantly lower probability to be in the zigzag channels. In a sense, silicalite becomes more and more "unidimensional" with increasing length of the hydrocarbon.

It is interesting to investigate the conformations of the alkanes in the various channels of silicalite in more detail. The alkane conformations were analyzed at intervals of 50 Monte Carlo cycles. The total number of analyzed configurations was 20000 . In Figures 9 and 10, the distribution of dihedral angles is shown for butane and dodecane, respectively. They are subdivided depend-
TABLE 9: Percentage of All-Trans Conformers of the n-Alkanes in Various the Channels in Silicalite $(T=298 \mathrm{~K})$

\begin{tabular}{ccclccc} 
straight & zigzag & intersection & & straight & zigzag & intersection \\
\hline $78_{1}$ & $84_{1}$ & $67_{2}$ & $\mathrm{C}_{9}$ & $60_{1}$ & $11_{1}$ & 0 \\
$63_{1}$ & $63_{1}$ & $55_{2}$ & $\mathrm{C}_{10}$ & $66_{1}$ & $9_{4}$ & 0 \\
$58_{2}$ & $49_{4}$ & $22_{4}$ & $\mathrm{C}_{11}$ & $68_{1}$ & $8_{2}$ & 0 \\
$52_{2}$ & $37_{3}$ & $5_{2}$ & $\mathrm{C}_{12}$ & $68_{1}$ & $7_{2}$ & 0 \\
$56_{1}$ & $26_{4}$ & 0 & & & &
\end{tabular}

ing on the section of the zeolite the molecule was located in. The symmetry of the curves indicates that the simulations were reliable. For dodecane the probability of finding a molecule in the intersection is small, and therefore the distribution of the dihedral angles in these sections of the zeolite show more scatter than the ones for the straight channels.

For butane, the distributions of the dihedral angle in the straight and zigzag channels are nearly identical. Most of the bonds are in the trans configurations. In the intersection the number of trans bonds is larger than the number of gauche bonds, but compared to the zigzag and straight channel the number of gauche bonds is larger. The channels with a diameter of $\approx 5.5 \AA$ favor the linear all-trans form of butane. In the intersection, with a diameter of $\approx 9 \AA$, the molecules have more space and can have more gauche bonds. For dodecane (Figure 10) the average number of gauche bonds along the chain in the straight channels is much smailer than that in the zigzag channels and intersection.

In Table 9, the conformations of the molecules are expressed in terms of the percentage of all-trans conformers. If an alkane is in the all-trans state, the molecule is perfectly linear. A gauche bond causes a deviation of this linear conformation. In a liquid alkane one expects the occurrence of a gauche bond with a certain probability. As a result, the percentage of all-trans chains decreases with increasing carbon number. The confinement of molecules in a zeolite changes this trend drastically. In the straight channels of silicalite, the number of trans conformers decreases with carbon number for the short chains as in the liquid phase. However, for the larger alkanes, starting with octane, the number of all-trans conformations increases with increasing carbon number. Apparently, a deviation of the linear conformation makes it increasingly difficult to fit into the channel. In Figure 11, the probability of a gauche bond as a function of the carbon-carbon bond is plotted. This figure shows that if a dodecane molecule has a gauche bond it is more likely to be at the end or beginning of the chain.

For the zigzag channels a different trend is observed. The number of all-trans conformations decreases continuously with carbon number. For the long alkanes most of the conformations in the zigzag channel will have at least one gauche defect. These gauche bonds occur with the same probability for every bond.

In the intersection, it is impossible to accommodate an alkane longer than octane in the all-trans state, and therefore most of the chains will have several gauche bonds (for example, for decane $36 \%$ of the molecules have 3 or more gauche bonds and for dodecane $23 \%$ of the molecules have more than 3 gauche bonds). In the intersections the larger alkanes will be in a hightly "coiled" state.

The distribution of the linear alkanes over the various channels is of importance to understand the details of adsorption and diffusion in silicalite. At present, there is no consensus in the literature on the distribution of the linear alkanes over the various channels. To interpret the experimental adsorption isotherms Jacobs et al. ${ }^{49}$ assumed that for $\mathrm{C}_{3}-\mathrm{C}_{5}$ there is no preferential adsorption, that $\mathrm{C}_{6}-\mathrm{C}_{8}$ favor the straight channels, and that for $\mathrm{C}_{9}-\mathrm{C}_{10}$ the zigzag channels are the preferred sites. Caro et al..$^{50}$ found the diffusion of short alkanes to be consistent with a model in which the alkanes preferentially adsorb in the intersections. Richards and Rees ${ }^{45}$ explained adsorption isotherms assuming that the longer alkanes prefer the zigzag channels. All these studies agree on preferential adsorption of alkanes taking place 


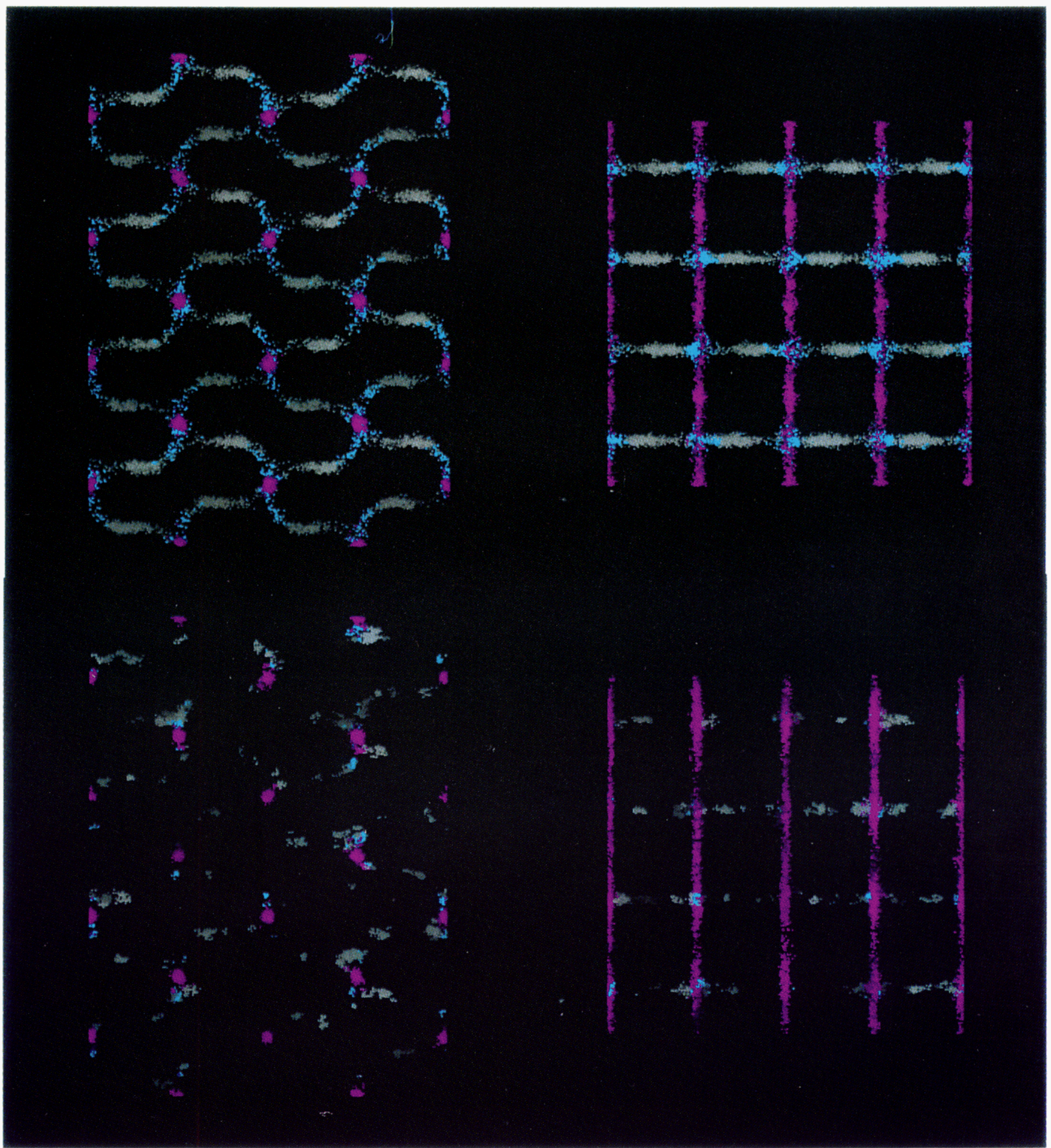

Figure 8. Distribution of butane (top) and dodecane (bottom) over the various channels of silicalite. At regular intervals during the simulations, the end to end vector of the alkane molecule is calculated and depending on its orientation a color is selected-blue for the intersection, gray for the zigzag channel, and magenta for the straight channel-and a dot in the selected color is drawn at the position of the center of mass of the molecule. The density of the dots is a measure of the probability of finding an alkane in a particular section of the zeolite. The left figures are projections on the $x-z$ plane, and the right figures projections on the $y-z$ plane (see Figure 6).

but disagree on which sites are favored by which alkanes. In contrast to this, Thamm ${ }^{33}$ concluded that his microcalorimetry experiments were consistent with a model without any preferential adsorption.

The siting of alkanes has also been studied using computer simulations. Also these studies did not yield a consistent picture. Titiloye et al. ${ }^{51}$ used energy minimization techniques to identify the positions of lowest energy. From these studies, Titiloye et al. concluded that the alkanes prefer the intersections and that the zigzag channels provide a less favorable energy than the straight channels. Note that the energy minimization technique assumes
$T=0 \mathrm{~K}$, and therefore the entropy of the alkanes was ignored. Molecular dynamics simulations - which do take entropy into account correctly-have been performed by June et al. ${ }^{5}$ and Nicholas et al. ${ }^{52}$ June et al. ${ }^{5}$ showed that $\mathrm{C}_{4}$ and $\mathrm{C}_{6}$ are equally likely to be found in the zigzag and straight channels and less likely to be in the intersection. The molecular dynamics simulations of $\mathrm{C}_{3}$ by Nicholas et al. ${ }^{52}$ showed a preferential adsorption in the zigzag channels. In summary, the issue of siting of hydrocarbons in silicalite must be regarded as far from resolved.

We can use our simulations to shed some light on the apparent confusion about the siting of the $n$-alkanes. For the short alkanes, 


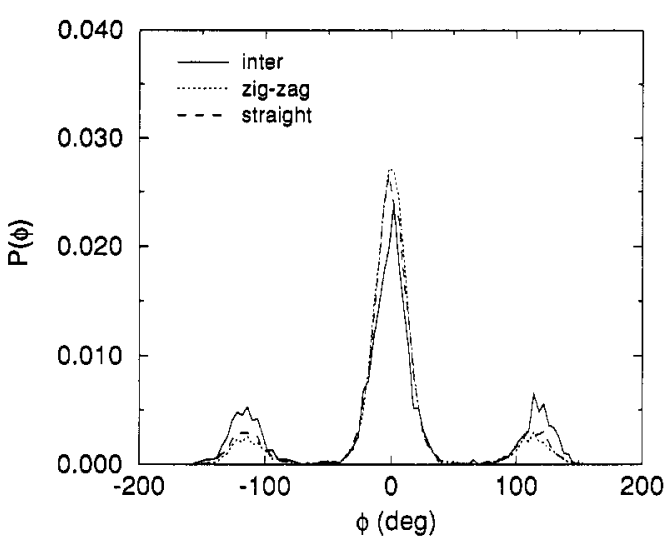

Figure 9. Distribution of the dihedral angle of butane in the various channels of silicalite.

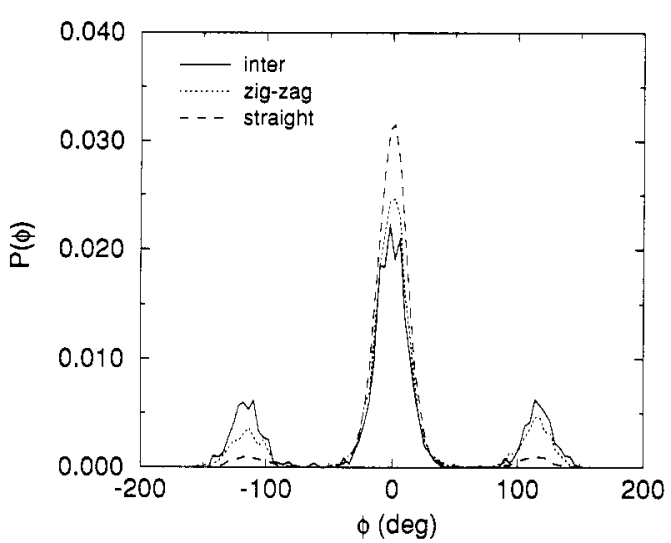

Figure 10. Distribution of the dihedral angles of dodecane in the various channels of silicalite.

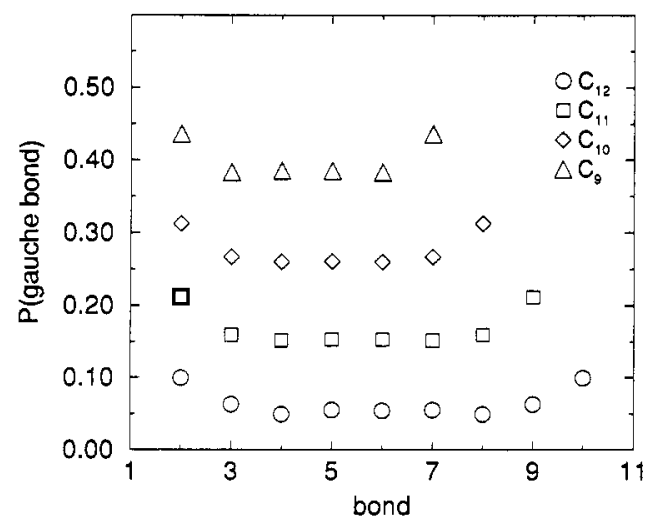

Figure 11. Probability of a gauche bond along the chain. Note that the curves are shifted by 0.1 for undecane, by 0.2 for decane, and by 0.3 for nonane.

our data are in perfect agreement with the simulations of June et al., 5 i.e., the straight and zigzag channel are equally likely and preferred above the intersections. For the larger alkanes, however, the analysis of the conformations shows that alkanes longer than octane need to have conformational defects to fit into the zigzag channels. Furthermore, the molecules have to be partly in the intersections because the length of the zigzag channel is about equal to or shorter than the length of the alkane. Apparently this is an unfavorable situation, and hence the longer molecules prefer the straight channels.

The energetics of hydrocarbon adsorption in the various channels is different. Therefore this preferential adsorption will influence the heats of adsorption which is found back in the observed change in slope of the calculated heats of adsorption as a function of carbon number (see Figure 4).

B. Mordenite. In Figure 12, the heats of adsorption of the

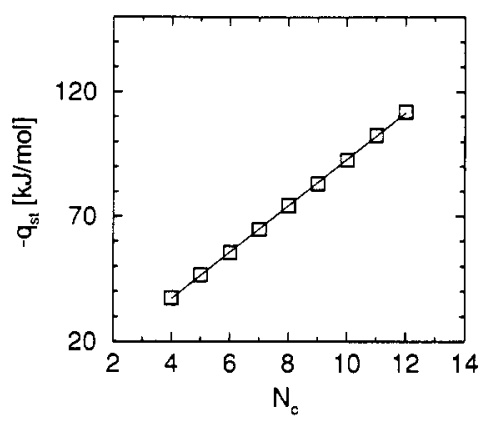

Figure 12. Heats of adsorption of the $n$-alkanes in mordenite. The solid line is a linear fit to the data, which give a slope of $9.3 \mathrm{~kJ} / \mathrm{mol}$ per carbon atom.

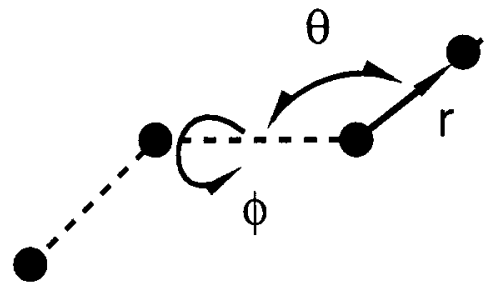

Figure 13. Definition of the bond length $r$, bond angle $\theta$, and torsional angle $\phi$.

alkanes in mordenite are presented. The heat of adsorption increases by $9.3 \mathrm{~kJ} / \mathrm{mol}$ per $\mathrm{CH}_{2}$ group. The heat of adsorption of a given alkane in mordenite is smaller than that in silicalite, as can be expected from the difference in pore size. Mordenite has wider pores $(7 \AA)$ than silicalite $(5.5 \AA)$, and therefore an alkane adsorbed in silicalite has more interactions with the walls..$^{53}$ We have not found experimental data to compare our results to.

\section{Concluding Remarks}

In this article, it is shown that the configurational-bias Monte Carlo method can be used to study the adsorption of the $n$-alkanes in various zeolites. Whereas conventional methods are limited to the short alkanes, this method allows us to simulate the adsorption of alkanes ranging from $\mathrm{C}_{4}$ to $\mathrm{C}_{12}$.

The calculated heats of adsorption for the small alkanes $\left(\mathrm{C}_{4}-\right.$ $\mathrm{C}_{8}$ ) in silicalite are found to be in very good agreement with experimental data. In addition, the simulations predict that the heats of adsorption of the alkanes in silicalite depend on the chain length. It would be interesting to verify these predictions experimentally.

In the future, we plan to extend this work to different types of hydrocarbons such as branched alkanes or alkenes and to mixtures. In addition, we will combine the configurational-bias Monte Carlo method with the grand-canonical ensemble. This will allow the calculation of adsorption isotherms of mixtures of hydrocarbons in arbitrary zeolites.

\section{Appendix A: Generation of Trial Orientations}

In this appendix, it is shown how the trial orientations in the configurational-bias Monte Carlo scheme are generated.

Let us first consider the general case with flexible bond length, bond bending, and torsion. The probability that we generate a trial configuration $\mathbf{b}$ is given by

$$
P(\mathbf{b}) \mathrm{db}=\frac{\exp \left[-\beta u^{\mathrm{int}}(\mathbf{b})\right] \mathrm{db}}{C}
$$

where $C$ is a normalization constant which is defined by

$$
C \equiv \int_{b} \mathrm{~d} \mathbf{b} \exp \left[-\beta u^{\text {int }}(\mathbf{b})\right]
$$

Note that in the configurational-bias Monte Carlo scheme we do not have to calculate this constant. 
It is convenient to represent the position of a atom using the bond length $r$, bond angle $\theta$, and torsional angle $\phi$ (see Figure 13). With these coordinates the volume element $\mathrm{db}$ is given by

$$
\mathrm{db}=r^{2} \cos (\theta) \mathrm{d} r \mathrm{~d} \theta \mathrm{d} \phi
$$

The internal energy is the sum of the bond vibration potential, the bond-bending potential, and the torsion potential:

$$
u^{\text {int }}(r, \theta, \phi)=u_{\text {bond-vib }}(r)+u_{\text {bending }}(\theta)+u_{\text {torsion }}(\phi)
$$

Substitution of eqs A4 and A3 into eq Al gives

$$
\begin{aligned}
& P(\mathbf{b}) \mathrm{db}=P(r, \theta, \phi) r^{2} \mathrm{~d} r \mathrm{~d} \theta \mathrm{d} \phi \\
&=\exp \left[-\beta u_{\text {bond-vib }}(r)\right] r^{2} \mathrm{~d} r \times \\
& \exp \left[-\beta u_{\text {bending }}(\theta)\right] \cos (\theta) \mathrm{d} \theta \exp \left[-\beta u_{\text {torsion }}(\phi)\right] \mathrm{d} \phi
\end{aligned}
$$

In our simulations we have used an alkane model with fixed bond length, and therefore in our case the first term in eq $A 5$ is a constant.

For the second carbon atom there are no internal interactions other than the constraints of the bond length. The distribution of trial orientations, eq 4 reduces to

$$
P_{2} \text { (b) } \mathrm{db} \propto \cos (\theta) \mathrm{d} \theta \mathrm{d} \phi
$$

Hence, the trial orientations are randomly distributed on the surface of a sphere. The algorithm that we have used for generating random vectors on the surface of a sphere is described in ref 12.

For the third atom, the internal energy contains the bondbending energy as well. This gives for the distribution of trial orientations

$$
P_{3}(\mathbf{b}) \mathrm{db} \propto \exp \left[-\beta u_{\text {bending }}(\theta)\right] \cos (\theta) \mathrm{d} \theta \mathrm{d} \phi
$$

where $u_{\text {bending }}(\theta)$ is given by eq 2 . To generate $k$ trial orientations that are distributed according to eq $\mathrm{A} 7$, we generate again a random vector on a unit sphere and determine the angle $\theta$. This vector is accepted with a probability $\exp \left[-\beta u_{\text {bending }}(\theta)\right]$. If rejected, this procedure is repeated until a value of $\theta$ has been accepted. In ref 28 it is shown that this acceptance/rejection method indeed gives a distribution of trial orientations given by eq A7. Note that the term $\cos \theta$ is taken into account by generating a random vector on a sphere. In this way, $k$ (or $k-1$ for the case of the old conformation) trial orientations are generated.

An alternative scheme would be to generate the angle $\theta$ uniformly $(\in[0, \pi])$ and to calculate the bond-bending energy corresponding to this angle. This angle $\theta$ is accepted with a probability $\cos (\theta) \exp \left[-\beta u_{\text {bending }}(\theta)\right]$. If rejected, this procedure is repeated until a value of $\theta$ has been accepted. The selected value of $\theta$ is supplemented with a randomly selected angle $\phi$. These two angles determine a new trial orientation.

For the fourth and higher carbon atoms, the internal energy includes both bond-bending and torsion energy. This gives for eq 4

$p_{l}^{\text {int }}(\mathbf{b}) \mathrm{db} \propto \exp \left[-\beta u_{\text {bending }}(\theta)\right] \exp \left[-\beta u_{\text {torsion }}(\phi)\right] \times$

$$
\cos (\theta) \mathrm{d} \theta \mathrm{d} \phi(\mathrm{A} 8)
$$

in which $u_{\text {torsion }}(\phi)$ is the torsion potential given by eq 3 . We again generate a random vector on a sphere and calculate the bond-bending angle $\theta$ and torsion angle $\phi$. These angles are accepted with a probability $\exp \left\{-\beta\left[u_{\text {bending }}(\theta)+u_{\text {torsion }}(\phi)\right]\right\}$. If these angles are rejected, new vectors are generated until one gets accepted.

Again the alternative scheme would be first to determine a bond-bending angle $\theta$ by generating $\theta$ uniformly on $[0, \pi]$ and calculating the bond-bending energy corresponding to this angle. This angle $\theta$ is then accepted with a probability $\cos (\theta)$ $\exp \left[-\beta u_{\text {bending }}(\theta)\right]$. This procedure is continued until we have accepted an angle. Next we generate a torsion angle randomly on $[0,2 \pi]$ and accept this angle with a probability $\exp \left[-\beta u_{\text {torsion }}(\phi)\right]$, again repeating this until a value has been accepted. In this scheme the bond angle and torsion angle are generated independently, which can be an advantage in cases where the corresponding potentials are sharply peaked.

\section{Appendix B: Proof of Correctness Sampling Scheme}

To prove that the sampling scheme used in this work is correct, i.e., a Boltzmann distribution of configurations is generated, we have to show that detailed balance is obeyed. Detailed balance implies that the rate of transformations from states o to states $n$ equals the reverse, or

$$
K(\mathrm{o} \rightarrow \mathrm{n})=K(\mathrm{n} \rightarrow \mathrm{o})
$$

The rate of transformations from $o$ to $n$ is the product of the probability of being in state 0 , the probability of generating configuration $\mathrm{n}$, and the acceptance of the move, or

$$
K(\mathrm{o} \rightarrow \mathrm{n})=\mathcal{N}(\mathrm{o}) P(\mathrm{o} \rightarrow \mathrm{n}) \operatorname{acc}(\mathrm{o} \rightarrow \mathrm{n})
$$

In case of a Boltzmann distribution, the probability of being in state $o$ is given by

$$
\mathcal{N}(0) \propto \exp \left(-\beta u^{\circ}\right)
$$

Metropolis Monte Carlo. It is instructive first to consider the ordinary metropolis Monte Carlo scheme. In this scheme a new configuration is generated by inserting a molecule at a random position with a random orientation. Hence, the probability of generating a particular configuration is equal for all configurations, and therefore the probability of generating configuration $\mathrm{n}$ is equal to the probability of generating configuration $\mathrm{o:}$

$$
P(\mathrm{o} \rightarrow \mathrm{n})=P(\mathrm{n} \rightarrow \mathrm{o})
$$

Substitution of eqs B3 and B4 into the condition of detailed balance (B1) gives

$$
\frac{\operatorname{acc}(o \rightarrow n)}{\operatorname{acc}(\mathrm{n} \rightarrow \mathrm{o})}=\frac{\exp \left(-\beta u^{\mathrm{n}}\right)}{\exp \left(-\beta u^{\circ}\right)}
$$

The acceptance rule for the (Metropolis) Monte Carlo scheme should obey this equation. One of the possible rules that obeys this equation is the traditional (Metropolis) acceptance rule

$$
\operatorname{acc}(\mathrm{o} \rightarrow \mathrm{n})=\min \left(1, \exp \left[-\beta\left(u^{\mathrm{n}}-u^{\circ}\right)\right]\right)
$$

Configurational-Bias Monte Carlo. In contrast to the Metropolis scheme, in the configurational-bias Monte Carlo scheme the probability of generating a particular configuration is different for each configuration. Therefore eq B4 is not valid and should be replaced. The probability of generating configuration $n$ is for each atom (except the first) the probability that we generate a particular trial direction (4) times the probability of selecting this direction (5),

$$
P(\mathrm{o} \rightarrow \mathrm{n})=\prod_{l=\frac{2}{M}}^{M} p_{l}^{\text {int }}\left(\mathbf{b}_{l}\right) p_{l}^{\text {ext }}\left(\mathbf{b}_{l}\right)
$$

The total energy of an alkane can be written as the sum of the internal and external energy:

$$
u=u^{\mathrm{ext}}+u^{\mathrm{int}}=\sum_{l=1}^{M}\left[u^{\mathrm{ext}}(l)+u^{\mathrm{int}}(l)\right]
$$

The internal energy is taken into account in the generation of the trial orientations, viz., eq 4 and the external interactions in the selection of a trial orientation, viz., eq 5 , which gives for eq $\mathrm{B} 7$

$$
P(\mathrm{o} \rightarrow \mathrm{n})=\prod_{l=2}^{M} \frac{\exp \left[-\beta u_{l}^{\mathrm{n}, \mathrm{int}}\left(\mathbf{b}_{j}\right)\right]}{C} \frac{\exp \left[-\beta u_{l}^{\mathrm{n}, \mathrm{ext}}\left(\mathbf{b}_{j}\right)\right]}{w^{\mathrm{n}, \mathrm{ext}}(l)}
$$




$$
=\exp \left(-\beta u^{n}\right) \frac{1}{C^{M-1}} \frac{1}{W^{m}}
$$

in which we have used eq 7 as the definition of $W^{\text {h }}$. Similarly, for the reverse move the probability of generating configuration $o$ can be written as

$$
P(\mathrm{n} \rightarrow 0)=\exp \left(-\beta u^{\circ}\right) \frac{1}{C^{M-1}} \frac{1}{W^{0}}
$$

Substitution of eqs B9 and B3 into eq B2 and imposing detailed balance (B1) gives for the ratio of the acceptance rules

$$
\begin{aligned}
\frac{\operatorname{acc}(o \rightarrow n)}{\operatorname{acc}(n \rightarrow o)} & =\frac{\exp \left(-\beta u^{n}\right) \exp \left(-\beta u^{\circ}\right)\left(1 / C^{M-1}\right)\left(1 / W^{\circ}\right)}{\exp \left(-\beta u^{0}\right) \exp \left(-\beta u^{n}\right)\left(1 / C^{M-1}\right)\left(1 / W^{n}\right)} \\
& =\frac{W^{n}}{W^{\circ}}
\end{aligned}
$$

Since eq 10 satisfies this condition, we have demonstrated that the above scheme obeys detailed balance, and hence conformations are generated with the correct (Boltzmann) weight. ${ }^{54}$ Note that the normalization constant $C$ introduced in eq 4 cancels in eq B11, and its numerical value is therefore not important in the configurational-bias Monte Carlo scheme.

\section{References and Notes}

(1) Maxwell, I. E.; Stork, W. H. J. In Introduction to Zeolite Science and Practice; Studies in Surface Science and Catalysis; Bekkum, H. van, Flaningen, E. M., Jansen, J. C. Eds.; Elsevier: Amsterdam, 1991.

(2) Chmelka, B. F.; Raftery, D.; McCormick, A. V.: Menorval, L. C. de Levine, R. D.; Pines, A. Phys. Rev. Lett. 1991, 66, 580-583.

(3) Kärger, J.; Ruthven, D. M. Diffusion in Zeolites and other Microporous Solids; John Wiley \& Sons: New York, 1992.

(4) Catlow, C. R. A., Ed. Modelling of Structure and Reactivity in Zeolites; Academic Press: London, 1992.

(5) June, R. L.; Bell, A. T.; Theodorou, D. N. J. Phys. Chem. 1992, 96 , 1051-1060.

(6) Pickett, S. D.; Nowak, A. K.; Thomas, J. M.; Peterson, B. K.; Swift, J. F.; Cheetham, A. K.; Ouden, C. J. J. den; Smit, B.; Post, M. J. Phys. Chem. $1990,94,1233-1236$

(7) Nowak, A. K.: Ouden, C. J. J. den; Pickett, S. D.; Smit, B.:Cheetham, A. K.; Post, M. F. M.; Thomas, J. M. J. Phys. Chem. 1991, 95, 848-854.

(8) Ouden, C. J. J. den; Smit, B.; Wielers, A. F. H.; Jackson, R. A.; Nowak, A. K. Mol. Simul. 1989, 4, 121-136.

(9) Siepmann, J. I.; Frenkel, D. Mol. Phys. 1992, 75, 59-70.

(10) Frenkel, D.; Mooij, G. C. A. M.; Smit, B. J. Phys.: Condens. Matter 1992, 4, 3053-3076.

(11) Smit, B.: Siepmann, J. I. Science 1994, 264, 1118-1120.

(12) Allen, M. P.; Tildesley, D. J. Computer Simulation of Liquids; Clarendon: Oxford, 1987.

(13) Ploeg, P. Van der; Berendsen, H. J. C. J. Chem. Phys. 1982, 76, $3271-3276$.

(14) Jorgensen, W. L.; Madura, J. D.; Swenson, C. J. J. Am. Chem. Soc. $1984,106,6638-6646$.

(15) Siepmann, J. I.; Karaborni, S.; Smit, B. Nature 1993, 365, 330-332. (16) Bezus, A. G.; Kiselev, A. V.; Lopatkin, A. A.; Du, P. Q. J. Chem. Soc., Faraday Trans. 2 1978, 74, 367-379.

(17) Demontis, P.; Suffritti, G. B.; Fois, E. S.; Quartieri, S. J. Phys. Chem. 1982, $96,1482-1490$.

(18) Harris, J.; Rice, S. A. J. Chem. Phys, 1988, 88, 1298-1306.

(19) Siepmann, J. I.; McDonald, I. R. Phys. Rev. Lett. 1993, 70, 453-456.

(20) Siepmann, J. I.; McDonald, I. R. Mol. Phys. 1993, 79, 457-473.
(21) Mooij, G. C. A. M.; Frenkel, D.; Smit, B. J.Phys.: Condens. Matter 1992, 4, L255-L259.

(22) Laso, M.; Pablo, J. J. de; Suter, U. W. J. Chem. Phys. 1992, 97, $2817-2819$.

(23) Siepmann, J. I.; Karaborni, S.; Smit, B. J. Am. Chem. Soc. 1993 $115,6454-6455$.

(24) Frenkel, D. Private communication.

(25) Wood, G. B.; Panagiotopoulos, A. Z.; Rowlinson, J. S. Mol. Phys. 1988, 63, 49-63.

(26) Barker, J. A.; Watts, R. O. Chem. Phys. Lett. 1969, 3, 144-145.

(27) June, R. L.; Bell, A. T.; Theodorou, D. N. J. Phys. Chem. 1990, 94, $1508-1516$.

(28) Press, W. H.; Flannery, B. P.; Teukolsky, S. A.; Vetterling, W. T. Numerical Recipes: The art of scientific computing, Cambridge University Press: Cambridge, 1986.

(29) Rychaert, J. P.; Bellemans, A. Chem. Phys. Lett. 1975, 30, 123-125.

(30) Smit, B. Computer Simulation of Phase Coexistence: from Atoms to Surfactants. Ph.D. thesis. Rijksuniversiteit Utrecht, The Netherlands, 1990.

(31) Smit, B. J. Chem. Phys. 1992, 96, 8639-8640.

(32) Note that in both the molecular dynamics and Monte Carlo simulations an interpolation technique was used to take into account the interactions with the zeolite.

(33) Thamm, H. Zeolites 1987, 7, 341-346.

(34) Stach, H.; Thamm, H.; Fiedler, K.; Schirmer, W. In New Developments in Zeolite Science and Technology; Proceedings of the 6th International Zeolite Conference; Olsen, D., Bisio, A., Eds.; Butterworth: Guildford, UK, 1984 , pp 225-231.

(35) Thamm, H.; Stach, H.; Schirmer, W.; Fahlke, B. Z. Phys. Chem. (Leipzig) 1982, 263, 461-469.

(36) Thamm, H.; Stach, H.; Fiebig, W. Zeolites 1983, 3, 95-97.

(37) Rakhmatkariev, G. U.; Zhalalov, Kh.R.; Akhmedov, K.S. Uzb.Khim. Zh. 1988, 3, 68-70.

(38) Dubinin, M. M.; Rakhmatkariev, G. U.; Isirikyan, A. A. Izv. Akad. Nauk SSSR, Ser. Khim. 1989, 10, 2333-2335.

(39) Stach, H.; Lohse, U.; Thamm, H.; Schirmer, W. Zeolites 1986, 6, 74-90.

(40) Flanigen, E. M.; Bennett, J. M.; Grose, R. W.; Cohen, J. P.; Patton,

R. L.; Kirchener, R. M.; Smith, J. V. Nature 1978, 271, 512-516.

(41) Abdul-Rehman, H. B.; Hasanain, M. A.; Loughlin, K. F. Ind. Eng. Chem. Res. 1990, 29, 1525-1535.

(42) Doelle, H.-J.; Heering, J.; Riekert, L.; Marosi, L. J. Catal. 1981, 71 $27-40$

(43) Hampson, J. A.; Rees, L. V. C. J. Chem. Soc., Faraday Trans. 1993, $89,3169-3176$

(44) Bülow, M.; Schodder, H.; Rees, L. V. C.; Richards, R. E. In New Developments in Zeolite Science and Technology; Proceedings of the 7th International Zeolite Conference; Murakami, Y., Iijima, A., Ward, J. W., Eds.: Elsevier: Amsterdam, 1986, pp 579-586.

(45) Richard, R. E.; Rees, L. V. C. Langmuir 1987, 3, 335-340.

(46) Kiselev, A. V.; Lopatkin, A. A.; Shulga, A. A. Zeolites 1985, 5 , $261-267$.

(47) Chiang, A. S.; Dixon, A. G.; Ma, Y. H. Chem. Eng. Sci. 1984, 39 1461-1468.

(48) Lechert, H.; Schweiter, W. In New Developments in Zeolite Science and Technology; Proceedings of the 6th International Zeolite Conference; Olsen, D., Bisio, A., Eds.; Butterworth: Guildford, UK, 1984, pp 210-216.

(49) Jacobs, P. A.; Beyer, H. K.; Valyon, J. Zeolites 1981, l, 161-168.

(50) Caro, J.; Bülow, M.; Schirmer, W.; Kärger, J.; Heink, W.; Pfeifer

H.: Zdanov, S. P. J. Chem. Soc., Faraday Trans. I 1985, 81 , 2541-2550.

(51) Titiloye, J. O.; Parker, S. C.; Stone, F. S.; Catlow, C. R. A. J. Phys. Chem. 1991, 95, 4038-4044.

(52) Nicholas, J. B.; Trouw, F. R.; Mertz, J. E.; Iton, L. E.; Hopfinger,

A. J. J. Phys. Chem. 1993, 97, 4149-4163.

(53) Smit, B.; Ouden, C. J. J. den. J. Phys. Chem. 1988, 92, 7169-7171.

(54) For clarity we have omitted in the proof some technical details. A complete proof can be found in ref 10 .

(55) Hufton, J. R.; Danner, R. P. AIChE J. 1993, 39, 954-961.

(56) Thamm, H.; Regent, N. I. Z. Chem. 1982, 22, 232-233. 